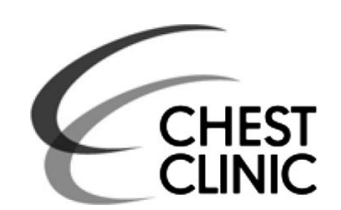

OPINION

\title{
Specialised commissioning for severe asthma: oxymoron or opportunity?
}

\author{
Binita Kane, ${ }^{1}$ Sophie Cramb $^{2}$ Val Hudson, ${ }^{2}$ Louise Fleming, ${ }^{3}$ Clare Murray, ${ }^{4}$ \\ John D Blakey ${ }^{5,6}$
}

${ }^{1}$ North West Lung Centre, University Hospital of South Manchester, Manchester, UK ${ }^{2}$ Asthma UK, London, UK ${ }^{3}$ Department of Paediatric Respiratory Medicine, National Heart and Lung Institute, Imperial College, London, UK

${ }^{4}$ Centre for Respiratory Medicine and Allergy, University of Manchester, Manchester, UK

${ }^{5}$ Department of Clinical Sciences, Liverpool School of Tropical Medicine, Liverpool, UK

${ }^{6}$ Department of Respiratory Medicine, Aintree University Hospital, Liverpool, UK

\section{Correspondence to}

Dr John Blakey, Department of Clinical Sciences, Centre for Tropical Infectious Diseases, Liverpool School of Tropical Medicine, Pembroke Place, Liverpool L5 3QA, UK : john.blakey@|stmed.ac.uk

Received 5 June 2015 Revised 25 September 2015 Accepted 21 October 2015

Published Online First

25 November 2015

\section{WHAT IS SPECIALISED COMMISSIONING?}

In the UK, almost $15 \%$ of the annual healthcare budget is set aside by the National Health Service (NHS) for specialised services. This significant resource ( $£ 14$ billion last year) is directed towards a clearly defined pool of uncommon and complex conditions, and specifically supports small numbers of 'centres of excellence' with large catchment areas. In respiratory medicine, specialised commissioning has funded services for cystic fibrosis and pulmonary hypertension for some years. However, variations in commissioning arrangements for other complex conditions have led to variable service provision. In 2013, NHS England (NHSE) produced service specifications for an additional set of respiratory conditions, including severe asthma ${ }^{1}$ (figure 1 ), and these have fuelled a good deal of debate.

\section{WHY SPECIALISED COMMISSIONING FOR ASTHMA?}

We all recognise asthma to be a major source of physical and psychological morbidity and of indirect and healthcare expenditure. Indeed, preventative inhaler therapy is the largest drug cost faced by the NHS. It came as little surprise to us that the recent National Review of Asthma Deaths revealed failings across the whole spectrum of asthma care, ${ }^{2}$ with this report and other publications (such as the NHS Atlas of Variation ${ }^{3}$ ) also highlighting disparities in outcomes across the country.

A small subset of asthmatics have severe, treatment-resistant disease. These individuals require expert, multidisciplinary team (MDT) assessment to comprehensively characterise their disease, evaluate psychological burden and tailor treatments, some of which may be high-cost therapies (such as monoclonal antibodies or bronchial thermoplasty) or treatment for complex issues such as vocal cord dysfunction or dysfunctional breathing. Current UK service provision is patchy, with some regions having multiple Trusts that run special-interest severe asthma clinics, with others having a single regional clinic for what maybe a similar-sized population. Furthermore, care within or between regions is not standardised.

We believe this current fragmented UK model of severe asthma care is not equitable for patients and is not sustainable clinically in view of the projected NHS funding gap. First, it is untenable for every UK Trust currently providing an 'asthma clinic' to develop and deliver a comprehensive specialist service with the finite resources available. Second, initial attempts to pool knowledge across severe asthma centres through the British Thoracic Society Severe Asthma Registry have further emphasised the benefits of sharing data and standardising procedures to improve outcomes ${ }^{45}$ and prevent overprescribing of expensive or toxic therapies. ${ }^{6}$ Third, increasing coordination of research across specialist centres affords a realistic opportunity to improve severe asthma management through large-scale investigator-led studies, such as the recent medical reaserch centre (MRC) supported UK Refractory Asthma Stratification (RASP-UK) programme. ${ }^{7}$ The ethos behind specialist commissioning is to provide equitable, high-quality care to patients with severe asthma in high-volume centres irrespective of postcode.

\section{BARRIERS TO SUCCESSFUL IMPLEMENTATION Who has severe asthma?}

Successful models of commissioning exist for services where end-users are readily identified either by the nature of the intervention (eg, one either has or has not had a lung transplant) or by the accepted objective threshold (eg, pulmonary hypertension). Severe asthma differs in that there is no single objective diagnostic test. Therefore, there is a considerable challenge in identifying from the millions of asthmatics, those who truly have treatment-resistant severe asthma rather than 'difficult asthma' due to physical and psychological comorbidity and environmental factors. This is a particular issue in areas where there remains an excessive reliance on reported symptoms rather than objective measures.

\section{The potential to exacerbate inequalities}

Since the release of the service specification, questions have been raised as to how a small number of centres could cater for all eligible patients across England. In other disease areas, centralised services increase the practical hardship of travel for patients ${ }^{89}$ and can be associated with delayed intervention. ${ }^{10}$ This appears to be a particular issue in geographically larger and less affluent regions. ${ }^{11}$ The increased practical or financial challenges of travelling to a specialist clinic may therefore discourage some individuals from accessing services in a timely and regular fashion.

\section{Who is special?}

In recent formal consultations and informal discussions, some healthcare professionals have expressed to the authors reservations over the perceived centralisation of 'specialised' services, and that some 


Primary care
Secondary care
- Criteria for referral to specialist centre ${ }^{4}$ : asthmatics with any of the following;
ventilation within the last 10 years.
- Continuous or frequent treatment with oral corticosteroids (defined as four or
more courses in the previous year).
- Fixed airflow obstruction, with a post bronchodilator forced expiratory volume
in 1 second (FEV $\mathrm{V}_{1}$ ) less than $70 \%$ of predicted normal.
- Referred as an adolescent transition patient from a paediatric severe asthma
service.

\begin{tabular}{|c|}
\hline Regional specialist centre of excellence \\
(Proposed 8-10 centres nationwide) \\
2-day intensive assessment by multidisciplinary team \\
\hline
\end{tabular}

2-day intensive assessment by multidisciplinary team

Follow-up
$\begin{aligned} & 70 \% \text { of patients to be followed up at specialist centre-all } \\ & \text { specialist treatment delivered at regional centre }\end{aligned}$
$30 \%$ likely alternative diagnosis and followed up
elsewhere

Figure 1 Under specialised commissioning, patients fitting the criteria would be referred to their most geographically convenient regional specialist unit from either primary, secondary or tertiary care. The specialist units, based at an unspecified number of pre-existing centres, would act as concentrated centres of excellence located around the UK, with all other severe asthma services decommissioned in their existing form. To be commissioned as a specialist unit, centres must have access to all facilities outlined in the specification ${ }^{4}$ required to deliver full multidisciplinary team assessments over 2-day case visits and must be led by at least two consultant respiratory physicians with a special interest in severe asthma.

have not engaged (or been engaged) in discussions. Although everyone wants to see the best service available to all patients, some feel there is insufficient evidence to support fully centralised care. There is particular concern about the potential effect on local services that have many years of experience of running an asthma clinic, on both the local population and the medical training. During discussions, those who run busy asthma services in district general hospitals have expressed a perception that key policy influencers tend to be academics from teaching hospitals who see a select group of patients away from the 'front line', and these individuals may not be representative of the wider 'difficult asthma' population. This is an interesting clash of viewpoints. We would argue that while it is clear that consultants in current specialist centres see a much higher volume of patients with severe asthma than their counterparts in district general hospitals, overall the burden of 'difficult' asthma is higher outside of specialist centres. Therefore, there is a requirement for expertise in the wider setting. However, this expertise should probably be complementary to that in specialist centres rather than attempting to widely 'upskill' physicians in nonspecialist hospitals: severe asthma is becoming ever more complex with advances in endotyping, new biomarkers becoming available and plethora of potentially costly biologics on the horizon. In order to maintain excellence in quality of care and financial sustainability, services may be better delivered in high-

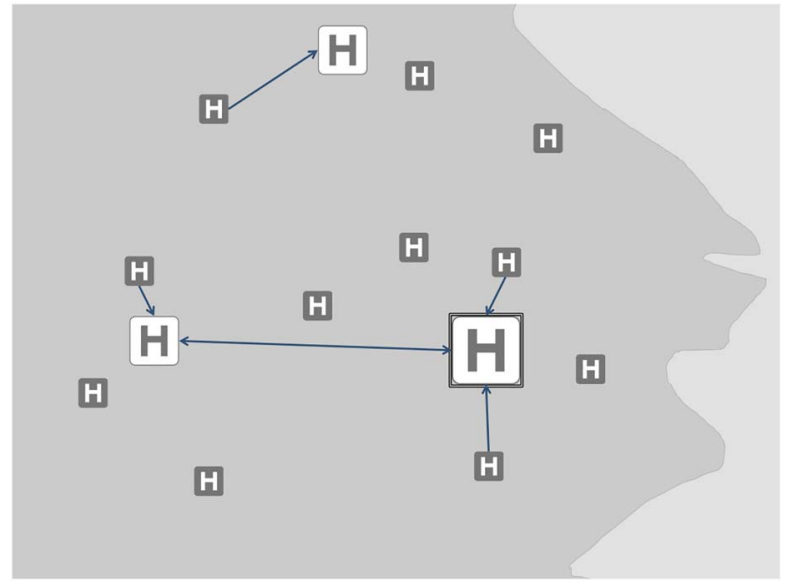

Figure 2 Example of a 'Hub and Network' model. The specialist, 'Hub', site refers to the centre which is commissioned to provide severe asthma services (depicted as largest white box). The wider network sites would supplement the specialised centre, rather than act as an alternative, and support the central service in delivering the best care for patients. The wider network might be made up of two types of sites: 'satellite' and 'peripheral' (depicted in white and grey, respectively). Satellite sites would be entrusted a level of responsibility to provide certain services governed by a contracted service level agreement with the Hub. The level of responsibility devolved would vary across networks and individual satellite sites depending on local needs and the expertise and capacity available at these sites.

volume specialist centres. These types of differences in opinion and viewpoints are inevitable when, over the years, independent local services have evolved in the absence of a national focus and create a political challenge in reorganising services to meet the specialised commissioning specification. Also inevitable is a degree of uncertainty around capacity and waiting times as the number of patients meeting the referral criteria could exceed expectations, particularly if the specialist units receive inappropriate referrals.

It should also be noted that specialist commissioning for severe asthma only applies to adults. Children with severe asthma will continue to be seen in a larger number of paediatric centres which operate as per the precommissioning model of adult severe asthma outlined above. In order to bridge this gap, all paediatric severe asthma services must be aligned to and work closely with a commissioned adult severe asthma service to ensure the smooth transition of care of young people (YP) with severe asthma to the right adult service. Not all YP will require transition to an adult severe asthma service; some may continue to be followed up in their local secondary care asthma clinic and some in primary care. The decision as to the most appropriate setting for ongoing care should be made jointly by the adult and paediatric teams.

\section{SPECIALISED COMMISSIONING AS AN OPPORTUNITY}

The specialised commissioning process represents a pivotal moment for asthma care in the UK. The conversations, collaborations and service developments associated with the process represent an opportunity to raise standards through the widespread adoption of standard protocols and procedures and through the sharing of data and expertise. Asthma clinicians from across the country recently presented NHSE with a consensus document, ${ }^{12}$ facilitated by Asthma UK, suggesting how this opportunity could be maximised to provide high-quality, accessible care to all patients while addressing local practical 
barriers to its implementation. This document argues that specialised commissioning can be effectively delivered though networked models of care similar to those that have proven to be effective for other problems (eg, stroke, burns and trauma).

Each area of England will need to develop their own service model for specialised commissioning depending on geography, local population and historical services. A proposed model is the 'Hub and Network' which involves a specialist unit commissioned to provide severe asthma services in line with the service specification (figure 2). This unit will support, and be supported by, surrounding primary and secondary care services. These network sites will agree to follow standard protocols for investigation, treatment and referral with 'common knowledge' that all participants are accountable for following agreed pathways. Management of patients fitting the criteria for specialist treatment will be facilitated by video-linked MDT discussion, in a similar model to lung cancer services, where assessment is carried out locally and treatment carried out in specialist centres after regional MDT discussion. These processes will ensure the gatekeeping of high-cost or toxic therapies, allow some compromise in opposing views on centralising services and deliver more patient-centred care through local services. Some sites will also be contracted to undertake clearly defined specialist activities to a predetermined, audited, high standard. In this way, patient experience and the needs of the local population can be prioritised and standardised. Specialist activity can be focused in centres of excellence rather than those of geographical convenience, while also recognising and rewarding expertise outside commissioned centres. A network approach enables service and outcome improvements through capturing and analysing performance data and through engagement with patients across the pathway. In the longer term, improvements will also be seen from the improved patient and clinical education structure and through networks facilitating clinical research. This might strike some as a naïve vision, but if there is not a collective and significant change in our approach to asthma management, outcomes will continue to be mediocre in relation to both other countries and the investment made by the NHS. Ensuring that children and adolescents with asthma have equitable access to specialised services is the next challenge.

\section{CONCLUSION}

There is tremendous potential to improve the care of people with severe asthma in the UK, and this could be catalysed by the specialised commissioning process. If we are to disperse excellence throughout the entire patient pathway, clinicians must retain a broad perspective and collegiate approach, while commissioners must acknowledge the complex environment in which they are operating. Excellence in severe asthma care and improved outcomes across the country will only be achieved if it is seamlessly integrated with collective improvements in all aspects of asthma services.

Twitter Follow Binita Kane at @binitakane

Acknowledgements Bernard Higgins and Marie Stolbrink gave feedback on the article; they are not included in the authorship but should be acknowledged.

Contributors BK and SC wrote the first draft of the article; this was edited and redrafted by JDB and VH. LF and CM contributed to the revision of the article to provide a paediatric perspective.

Competing interests $B K, L F, C M$ and JDB work in specialist asthma centres. SC was, at the time of writing, a 'Patient and Public Voice' member of the Respiratory Clinical Reference Group.

Provenance and peer review Commissioned; externally peer reviewed.

\section{REFERENCES}

1 NHS England. A14/S/b 2013/14 NHS Standard Contract for Respiratory: Severe Asthma (adult). http://www.england.nhs.uk/wp-content/uploads/2013/06/ a14-respiratory-sev-asthma.pdf (accessed 5 May 2015).

2 Royal College of Physicians. Why asthma still kills the National Review of Asthma Deaths (NRAD). Royal College of Physicians, 2014. https://www.rcplondon.ac.uk/ sites/default/files/why-asthma-still-kills-full-report.pdf (accessed 5 May 2015).

3 Public Health England. The atlas of variation of respiratory disease. Eastern Region Public Health Observatory, 2012. http://www.rightcare.nhs.uk/index.php/atlas/ respiratorydisease/ (accessed 5 May 2015.)

4 Gibeon D, Heaney LG, Brightling CE, et al. Dedicated severe asthma services improve healthcare utilisation and quality of life. Chest 2015;148:870-6.

5 Sweeney J, Brightling CE, Menzies-Gow A, et al, British Thoracic Society Difficult Asthma Network. Clinical management and outcome of refractory asthma in the UK from the British Thoracic Society Difficult Asthma Registry. Thorax 2012;67:754-6.

6 O'Neill S, Sweeney J, Patterson CC, et al. The cost of treating severe refractory asthma in the UK: an economic analysis from the British Thoracic Society Difficult Asthma Registry. Thorax 2014;706:14.

7 Refractory Asthma Stratification Programme. http://www.rasp.org.uk (accessed 24 Sep 2015.)

8 Payne $S$, Jarrett $N$, Jeffs $D$. The impact of travel on cancer patients' experiences of treatment: a literature review. Eur J Cancer Care (Engl) 2000;9:197-203.

9 McGrath P. Overcoming the distance barrier in relation to treatment for haematology patients: Queensland findings. Aust Health Rev. 2015;39: 344-50.

10 Massarweh NN, Chiang YJ, Xing Y, et al. Association between travel distance and metastatic disease at diagnosis among patients with colon cancer. J Clin Oncol 2014;32:942-8.

11 Cook PA, Downing J, Wheater CP, et al. Influence of socio-demographic factors on distances travelled to access HIV services: enhanced surveillance of HIV patients in north west England. BMC Public Health 2009;9:78.

12 Asthma UK. A Network-Based Approach for Specialised Severe Asthma Services A proposal to support specialised commissioning for adult severe asthma services. http://www.asthma.org.uk/advice-severe-asthma (accessed 3 Jun 2015). 